\title{
Condiciones y Medio Ambiente de Trabajo de los alumnos del ciclo profesional y de los graduados del plan vigente de las Ingenierias de la FaCENA UNNE. Conocimientos comple- mentarios requeridos por el mercado de trabajo de la región NEA Argentina.
}

Ing. Esp.Norberto Sanabria, Dr. Juan Nápoles Valdez, Ing. Guillermo Eduardo Langton, Ing. Emilio Fabián, Scozzina, Lic. Esp. Alicia Amarilla (*)

Resumen: Con este proyecto se busca indagar sobre las condiciones y medioambiente de trabjo, de los alumnos y de los graduados de los ultimos cinco años de las carreras de ingenierias de la FACENA, ademas de explorar sobre el perfil laboral que requieren las empresas y organizaciones del medio. Con la informacion obtenida se generará conocimeintos actualizado sobre la sitacion laboral, de los alunmos y profesioanles de esta disciplina en el NEA

Palabras claves: CyMAT, Insercion laboral, Ingenieros, Perfil Laboral.

E1 proyecto esta orientado a aportar sobre la satisfaccion de la necesidad del pais y de la region, la de contar con profesionales de la ingnieria. Actualemente, en el pais egresan de las carreras de ingenieria 8.040 ingenieros y según estimaciones del Consejo Federal de Decanos de Ingeniería, nuestro país necesitaría casi duplicar esta cifra para llegar al menos a un ingeniero cada 4.000 habitantes. Se considera a las carreras de ingeniería, son esenciales para fomentar el crecimiento de un país o región.

Los alumnos, incriptos en las carreras de ingenieria de la Facultad de Ciencias Exctas y Natuales y Agrimensura (FaCENA) de la Universidad Nacional del Nordeste (UNNE) provienen tanto de las

(*) Grupo de Telemedicinay Gestión de Recursos Humanos (Res 044/13CD) y PEPEI-UNNE-PEFI (Res 4655/14SPU) Contacto: norbertoargentinosanabria@gmail.com 
provincias del NEA, como de países limítrofes; llegan con diversas: costumbres, formacion de base y cierta predisposición por el estudio de las carreras tecnológicas.

Desde el Estado se establecieron diversos instrumentos de política activa, orientado en uno de sus ejes centrales, al logro del mejoramiento de indicadores académicos e incentivos para la finalización de la carrera de alumnos avanzados.

La formación academica de calidad incorporado a través de los contenidos; es fundamental para todos los profesional universitario. En el mercado laboral actual se demanda, además, de manera imperiosa y excluyente, graduados con competencias, actitudes y aptitudes que le permitan desempeñarse no sólo con alta capacitación técnica; sino que además; con valores y habilidades diferenciales propias de los profesionales de esta nueva era.

E1 mundo moderno y el mercado de trabajo, han modificado drásticamente las reglas que definen la actuación profesional. Obliga a los profesionales a adquirir aptitudes que les permitan desarrollarse en entornos inestables y altamente competitivos.

El nuevo ingeniero debe estar preparado para cambiar y crear, no sólo en lo que se refiere a sus trabajos o habilidades técnicas sino también en cuanto a su propio perfil y a las oportunidades de crecimiento profesional.

La formación académica basada en contenidos teóricos, prácticos y de laboratorios internos es indudablemente imprescindible; pero actualmente no se considera suficiente. En los perfiles de los ingenieros modernos se requieren, además otras aptitudes como creatividad, innovación, capacidad para trabajar en grupo multidisciplinario, conducir equipos exitosos, liderazgo y las habilidades de gestión, promotor de procesos de creación o innovación y como emprendedor de negocios basados en sus aptitudes técnicas.

Estas razones motivaron la necesidad de investigar con mayor profundidad la situación de los alumnos del último ciclo que se encuentra trabajando en el medio, en cuanto a las condiciones laborales y el impacto que esto provoca en la carrera universitaria y en la posibilidad de finalizar sus estudios.

El porcentaje de egreso de las tres carreras, requiere un análisis cuali-cuantitativo donde se conjuguen y valoren la diversidad de factores que pueden actuar como facilitadores u obstaculizadores del avance en la trayectoria final del estudiante.

Se puede observar una disminución del rendimiento académico en los últimos años de las carreras y unos de los factores que se estima es que los alumnos avanzados asumen mayores responsabilidades laborales, obstaculizando la finalizacion de las carreras.

Esta cuestión conlleva a preguntarnos ¿Cuáles son las condiciones laborales en la que están trabajando los alumnos del último ciclo, de las carreras de ingenira de la FaCENA- UNNE?

Sin embargo, tanto las instituciones gubernamentales, como las asociaciones profesionales de la región coinciden en 


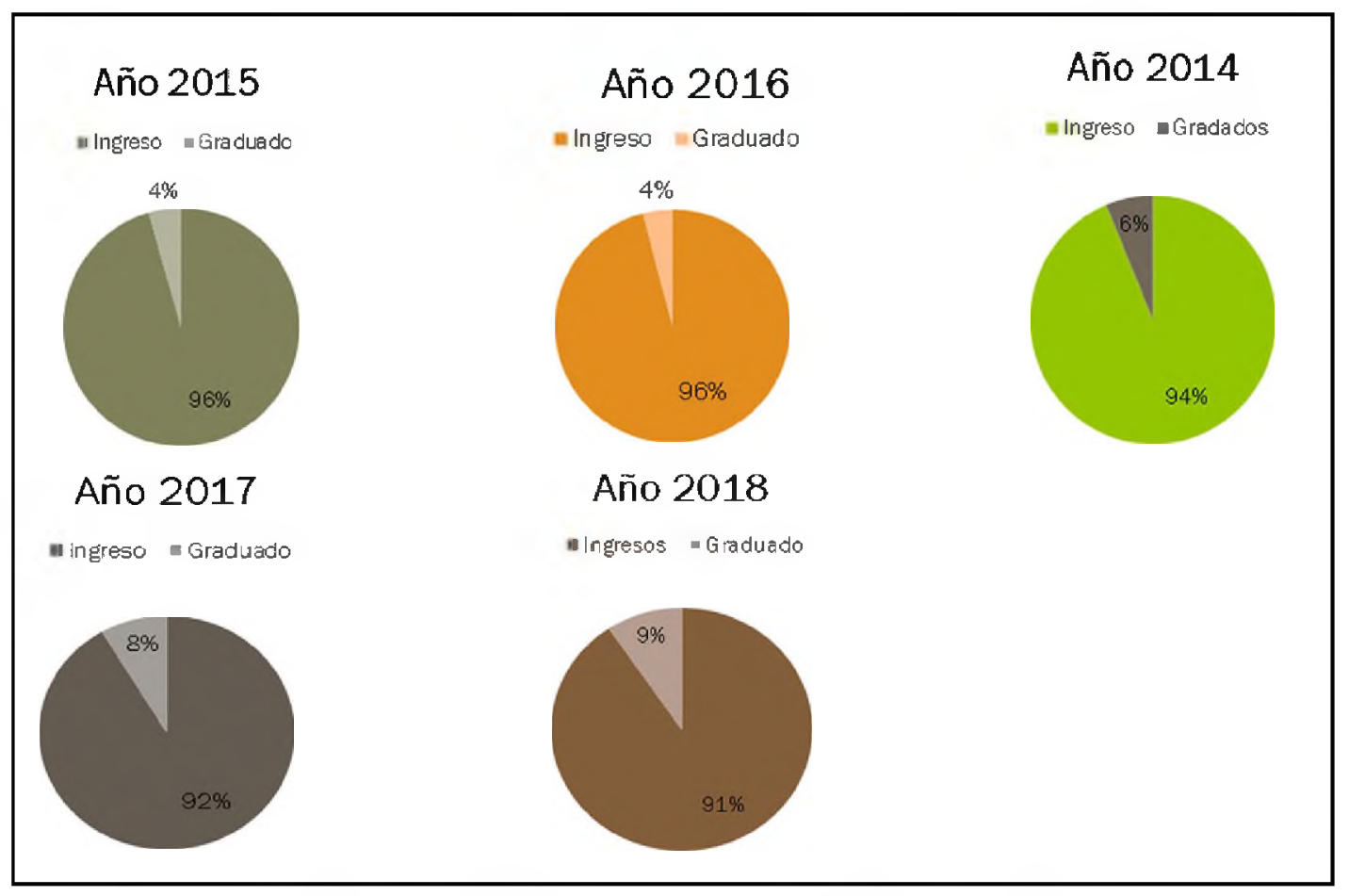

Figura 1: proyeccion de los ultimos 5 años, en realcion a los inscriptos y egredas de las carreras de ingenieria de la FaCENA-UNNE (fuente propia).

afirmar que existe una importante demanda insatisfecha para contar con ingenieros en sus planteles. Las pequeñas y medianas empresas y los emprendedores demandan la asistencia de ingenieros pero no encuentran profesionales preparados para relacionarse con el mundo de los negocios; este es un perfil altanamente demandado actualmente. Otras interrogantes que se plantean a partir de esta problemática es: ¿Estamos formando profesionales con el perfil requerido por el mercado de trabajo actual?

En base a todo lo planteado se considera relevante investigar sobre estas diversas problemáticas planteadas anteriormente y responder las preguntas formuladas en el trabajo de investigacion, con el propósito de alcanzar los objetivos trazados y aportar, a través de los resultados, propuestas concretas, para colaborar en la formación de ingenieros con actitudes y aptitudes altamente valoradas por el mercado de trabajo actual de la región.

Julio Cesar Neffa (1986) define que "las condiciones y medio ambiente de trabajo (CyMAT) están constituidas por los factores socio-técnicos y organizacionales del proceso de producción implantado en el establecimiento (o condiciones de trabajo) y por los factores de riesgo del medio ambiente de trabajo. Ambos grupos de factores constituyen las exigencias, requerimientos y limitaciones del puesto de trabajo, cuya articulación sinérgica o combinada da lugar a la carga global del trabajo prescripto, la 
cual es asumida, asignada o impuesta a cada trabajador, provocando de manera inmediata o mediata, efectos directos e indirectos, positivos o negativos, sobre la vida y la salud física, síquica $\mathrm{y} / \mathrm{o}$ mental de los trabajadores. Dichos efectos están en función de la actividad o trabajo efectivamente realizado, de las características personales, de las respectivas capacidades de adaptación y resistencia de los trabajadores ante los dos grupos de factores antes mencionados. Esos factores están determinados en última instancia por el proceso de trabajo vigente el cual a su vez es el resultante de las relaciones sociales y de la inter-relación entre las variables que actúan a nivel del contexto socio-económico y las características propias de los establecimientos. Es este proceso de trabajo el que define la naturaleza específica de la tarea a realizar por el colectivo de trabajo y por cada uno de los que ocupan dichos puestos".

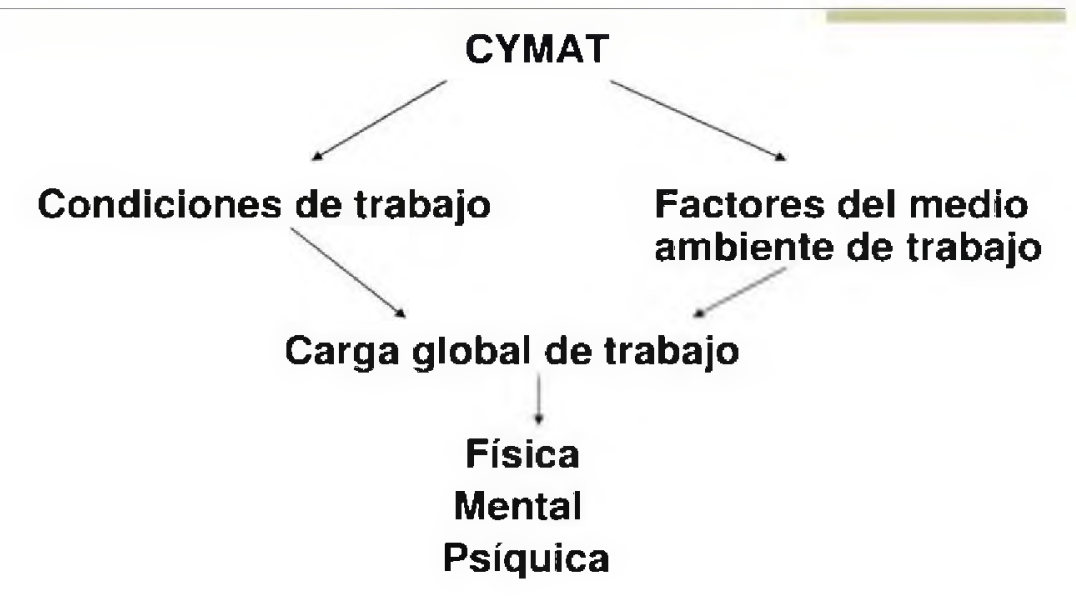

Figura 2: CyMAT

Se puede afirmar que la causa principal (aunque no la única) que determina las CyMAT es el proceso de trabajo, por medio de la organización y el contenido del trabajo. El proceso de trabajo genera determinantes del medio ambiente físico, emocionales y cognitivos, que constituyen demandas para ejecutar el trabajo prescripto y los trabajadores deben movilizar su fuerza de trabajo, su creatividad e involucrarse para hacer frente a los incidentes y a las deficiencias de la concepción para lleva a cabo la actividad, asumiendo riesgos y sufrimiento porque están dejando de lado o violando incluso las normas prescriptas.

Los riesgos para la salud provenientes del medio ambiente pueden ser físicos, químicos, biológicos, factores tecnológicos y de seguridad. Las condiciones de trabajo (resultantes de factores socio-técnicos y organizacionales) pueden compensar o intensificar dichos riesgos e impactan en 
la duración y configuración del tiempo de trabajo, la organización y el contenido del trabajo, el sistema de remuneración, el efecto del cambio científico y tecnológico y el aprendizaje en el uso de nuevas tecnologías; también, en la existencia o no de dispositivos de prevención de los riesgos ocupacionales, el acceso a las obras sociales y de bienestar ofrecidas por la empresa a sus trabajadores, el carácter o estatuto jurídico de la relación salarial y las posibilidades de que los trabajadores participen efectivamente en la identifica- ción de los riesgos, propongan medidas de prevención e intervengan en su ejecución y evaluación, si existen los comités mixtos de prevención de los riesgos. (Neffa, 1986).

Las fuertes restricciones físicas que impone el puesto de trabajo (como los esfuerzos físicos prolongados, carga y descarga de objetos pesados, trabajar en permanencia en posturas penosas y soportar vibraciones, situaciones que predominan en la industria, la construcción, el transporte y las actividades agropecuarias), representan un contexto agresivo debido.

\section{BIBLIOGRAFIA}

Moncada, S., Llorens, C., Kristensen, T. S. (2003) Método ISTAS21 COPSOQ. Manual para la evaluación de riesgos psicosociales en el trabajo. Barcelona: ISTAS 2003 (accesible en formato electrónico en www. istas.net).

Neffa, Julio Cesar (2015) Los Riesgos Psicosociales en el Trabajo. Contribución a su estudio. Libro Digital ISBN 978-987-215799-9.

Neffa, J. C. (1989) Qué son las condiciones y medio ambiente de trabajo? Propuesta de una nueva perspectiva. Ed. CEIL-CONICET, Ârea de Estudio e Investigación en Ciencias Sociales del Trabajo de la SECYT, CREDAL-URA N N $^{\circ} 11$ au CNRS, Ed. Hvmanitas, Buenos Aires.

Neffa, J. C. (Dir.), Cordone, H., Korinfeld, S., Giraudo, E. (1987) Condiciones y Medio Ambiente de Trabajo en la República Argentina. Tres volúmenes, Ed. CEIL-Hvmanitas, Buenos Aires.

Nieto, Héctor (1999) Epidemiología de los accidentes de trabajo entre los trabajadores sanitarios. Rev. Del Inst. de Higiene y Med. 
Social.

Rodríguez Gómez, Gregorio (1999) Metodología de la Investigación Cualitativa-Edición Alsives- Málaga.

Sabino, Carlos (1996) El proceso de Investigación- Editorial Humanitas, Bs As.

Vasilachis de Gialdino, Irene (2007) Estrategia de Investigación Cualitativo- Editorial Gedisa. Bs As.

Verón, O.E. (2003) Lineamientos Estratégicos. Superintendencia de Riesgos de Trabajo. Ed. SRT. Bs As.

Villate, R. (1990) El método del árbol de causas (para analizar los accidentes de trabajo en vistas a su prevención). Ed. Área de
Estudios e Investigaciones Laborales de la SECYT, CEIL-CONICET, CREDALCNRS, Hvmanitas.

Volkoff, S. (1993) Estadísticas sobre condiciones y medio ambiente de trabajo. Métodos y resultados. Ed. Asociación Trabajo y Sociedad, Buenos Aires.

Wisner, A. (1988) Ergonomía y Condiciones de Trabajo. Editorial Hvmanitas.

http://www.diarionorte.com/article/139478/ argentina-imperiosa-demanda-de-ingenieros-

http://www.empleos.clarin.com/postulantes/ avisos/trabajo/RE1411 


\section{EJE 3}

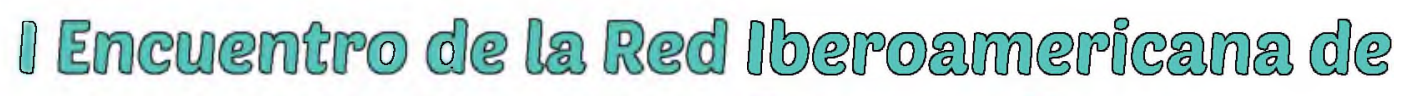
Unvestigacion, Desappollo y

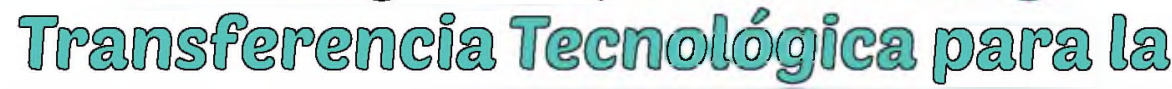

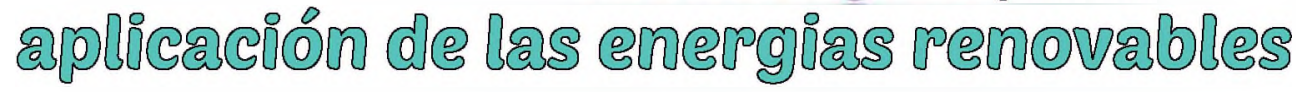
y cuid@do del ฌmbiente.

\section{Ribera

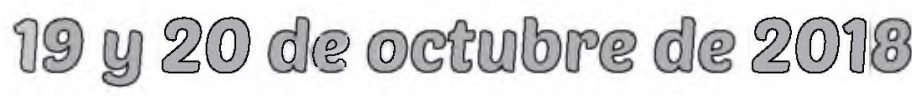

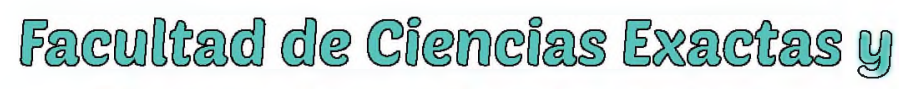

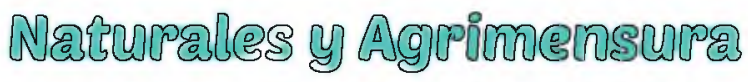

$\checkmark$

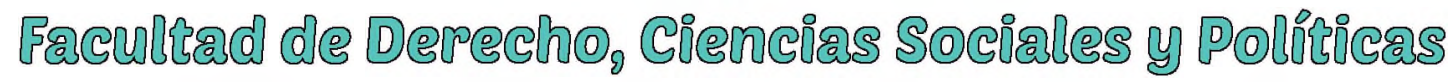

UNDVERSIDAD NACIONAL DEL NORDESTE

CORRDENTES- ADGENTUNA 
